Bull. Austral. Math. Soc.

VOL. 69 (2004) [327-340]

\title{
ON BESSEL AND GRÜSS INEQUALITIES \\ FOR ORTHORNORMAL FAMILIES IN INNER PRODUCT SPACES
}

\section{S.S. DRAGomiR}

A new reverse of Bessel's inequality for orthornormal families in real or complex inner product spaces is obtained. Applications for some Grüss type results are also provided.

\section{INTRODUCTION}

In the recent paper [2], the following refinement of the Grüss inequality has been proved:

THEOREM 1. Let $(H,\langle\cdot, \cdot))$ be an inner product space over $\mathbb{K}(\mathbb{K}=\mathbb{R}, \mathbb{C})$ and $e \in H,\|e\|=1$. If $\phi, \Phi, \gamma, \Gamma$ are real or complex numbers and $x, y$ are vectors in $H$ such that either

$$
\operatorname{Re}\langle\Phi e-x, x-\phi e\rangle \geqslant 0 \text { and } \operatorname{Re}\langle\Gamma e-y, y-\gamma e\rangle \geqslant 0
$$

or, equivalently,

$$
\left\|x-\frac{\phi+\Phi}{2} e\right\| \leqslant \frac{1}{2}|\Phi-\phi|, \quad\left\|y-\frac{\gamma+\Gamma}{2} e\right\| \leqslant \frac{1}{2}|\Gamma-\gamma|
$$

hold, then we have the following refinement of the Grüss inequality

$$
\begin{aligned}
\mid\langle x, y\rangle & -\langle x, e\rangle\langle e, y\rangle \mid \\
& \leqslant \frac{1}{4}|\Phi-\phi||\Gamma-\gamma|-[\operatorname{Re}\langle\Phi e-x, x-\phi e\rangle]^{1 / 2}[\operatorname{Re}\langle\Gamma e-y, y-\gamma e\rangle]^{1 / 2} \\
& \leqslant \frac{1}{4}|\Phi-\phi||\Gamma-\gamma| .
\end{aligned}
$$

The constant $1 / 4$ is best possible in both inequalities.

Note that the inequality between the first and last term in (1.3) was first established in $[1]$.

A generalisation of the above result for finite families of orthornormal vectors has been pointed out in [3].

Received 3rd November, 2003

Copyright Clearance Centre, Inc. Serial-fee code: 0004-9727/04 \$A2.00+0.00. 
THEOREM 2. Let $\left\{e_{i}\right\}_{i \in I}$ be a family of orthornormal vectors in $H, F$ a finite part of $I, \phi_{i}, \Phi_{i}, \gamma_{i}, \Gamma_{i} \in \mathbb{K}(\mathbb{K}=\mathbb{R}, \mathbb{C}), i \in F$ and $x, y \in H$. If

$$
\begin{gathered}
\operatorname{Re}\left\langle\sum_{i \in F} \Phi_{i} e_{i}-x, x-\sum_{i \in F} \phi_{i} e_{i}\right\rangle \geqslant 0 \\
\operatorname{Re}\left\langle\sum_{i \in F} \Gamma_{i} e_{i}-y, y-\sum_{i \in F} \gamma_{i} e_{i}\right\rangle \geqslant 0
\end{gathered}
$$

or, equivalently,

$$
\begin{aligned}
\left\|x-\sum_{i \in F} \frac{\Phi_{i}+\phi_{i}}{2} e_{i}\right\| & \leqslant \frac{1}{2}\left(\sum_{i \in F}\left|\Phi_{i}-\phi_{i}\right|^{2}\right)^{1 / 2}, \\
\left\|y-\sum_{i \in F} \frac{\Gamma_{i}+\gamma_{i}}{2} e_{i}\right\| & \leqslant \frac{1}{2}\left(\sum_{i \in F}\left|\Gamma_{i}-\gamma_{i}\right|^{2}\right)^{1 / 2},
\end{aligned}
$$

hold, then we have the inequalities

$$
\begin{aligned}
\mid\langle x, y\rangle & -\sum_{i \in F}\left\langle x, e_{i}\right\rangle\left\langle e_{i}, y\right\rangle \mid \\
\leqslant & \frac{1}{4}\left(\sum_{i \in F}\left|\Phi_{i}-\phi_{i}\right|^{2}\right)^{1 / 2} \cdot\left(\sum_{i \in F}\left|\Gamma_{i}-\gamma_{i}\right|^{2}\right)^{1 / 2} \\
& \quad-\left[\operatorname{Re}\left\langle\sum_{i \in F} \Phi_{i} e_{i}-x, x-\sum_{i \in F} \phi_{i} e_{i}\right\rangle\right]^{1 / 2}\left[\operatorname{Re}\left\langle\sum_{i \in F} \Gamma_{i} e_{i}-y, y-\sum_{i \in F} \gamma_{i} e_{i}\right\rangle\right]^{1 / 2} \\
\leqslant & \frac{1}{4}\left(\sum_{i \in F}\left|\Phi_{i}-\phi_{i}\right|^{2}\right)^{1 / 2} \cdot\left(\sum_{i \in F}\left|\Gamma_{i}-\gamma_{i}\right|^{2}\right)^{1 / 2} \cdot
\end{aligned}
$$

The constant $1 / 4$ is best possible.

REMARK 1. We note that the inequality between the first term and the last term for real inner products under the assumption (1.4) has been proved by Ujević in [4].

The following corollary provides a reverse for the well known Bessel's inequality in real or complex inner product spaces (see also [3]).

Corollary 1. With the above assumptions for $\left\{e_{i}\right\}_{i \in I}, F, \phi_{i}, \Phi_{i}$ and $x$, one has the inequalities

$$
\begin{aligned}
0 & \leqslant\|x\|^{2}-\sum_{i \in F}\left|\left\langle x, e_{i}\right\rangle\right|^{2} \\
& \leqslant \frac{1}{4} \sum_{i \in F}\left|\Phi_{i}-\phi_{i}\right|^{2}-\operatorname{Re}\left\langle\sum_{i \in F} \Phi_{i} e_{i}-x, x-\sum_{i \in F} \phi_{i} e_{i}\right\rangle \\
& \leqslant \frac{1}{4} \sum_{i \in F}\left|\Phi_{i}-\phi_{i}\right|^{2},
\end{aligned}
$$

with $1 / 4$ as the best possible constant in both inequalities. 
The main aim of this paper is to point out a different reverse for the Bessel and Grüss inequalities stated above. Some related results are also outlined.

\section{A REVERSE OF BESSEL'S INEQUALITY}

The following lemma holds.

LEMma 1. Let $\left\{e_{i}\right\}_{i \in I}$ be a family of orthornormal vectors in $H, F$ a finite part of $I, \lambda_{i} \in \mathbb{K}, i \in F, r>0$ and $x \in H$. If

$$
\left\|x-\sum_{i \in F} \lambda_{i} e_{i}\right\| \leqslant r
$$

then we have the inequality

$$
0 \leqslant\|x\|^{2}-\sum_{i \in F}\left|\left\langle x, e_{i}\right\rangle\right|^{2} \leqslant r^{2}-\sum_{i \in F}\left|\lambda_{i}-\left\langle x, e_{i}\right\rangle\right|^{2}
$$

Proof: Consider

$$
\begin{aligned}
I_{1} & :=\left\|x-\sum_{i \in F} \lambda_{i} e_{i}\right\|^{2}=\left\langle x-\sum_{i \in F} \lambda_{i} e_{i}, x-\sum_{j \in F} \lambda_{j} e_{j}\right\rangle \\
& =\|x\|^{2}-\sum_{i \in F} \lambda_{i} \overline{\left\langle x, e_{i}\right\rangle}-\sum_{i \in F} \overline{\lambda_{i}}\left\langle x, e_{i}\right\rangle+\sum_{i \in F} \sum_{j \in F} \lambda_{i} \overline{\lambda_{j}}\left\langle e_{i}, e_{j}\right\rangle \\
& =\|x\|^{2}-\sum_{i \in F} \lambda_{i} \overline{\left\langle x, e_{i}\right\rangle}-\sum_{i \in F} \overline{\lambda_{i}}\left\langle x, e_{i}\right\rangle+\sum_{i \in F}\left|\lambda_{i}\right|^{2}
\end{aligned}
$$

and

$$
\begin{aligned}
I_{2} & :=\sum_{i \in F}\left|\lambda_{i}-\left\langle x, e_{i}\right\rangle\right|^{2}=\sum_{i \in F}\left(\lambda_{i}-\left\langle x, e_{i}\right\rangle\right)\left(\overline{\lambda_{i}}-\overline{\left\langle x, e_{i}\right\rangle}\right) \\
& =\sum_{i \in F}\left[\left|\lambda_{i}\right|^{2}+\left|\left\langle x, e_{i}\right\rangle\right|^{2}-\overline{\lambda_{i}}\left\langle x, e_{i}\right\rangle-\lambda_{i} \overline{\left\langle x, e_{i}\right\rangle}\right] \\
& =\sum_{i \in F}\left|\lambda_{i}\right|^{2}+\sum_{i \in F}\left|\left\langle x, e_{i}\right\rangle\right|^{2}-\sum_{i \in F} \overline{\lambda_{i}}\left\langle x, e_{i}\right\rangle-\sum_{i \in F} \lambda_{i} \overline{\left\langle x, e_{i}\right\rangle}
\end{aligned}
$$

If we subtract $I_{2}$ from $I_{1}$ we deduce an identity that is interesting in its own right

$$
\left\|x-\sum_{i \in F} \lambda_{i} e_{i}\right\|^{2}-\sum_{i \in F}\left|\lambda_{i}-\left\langle x, e_{i}\right\rangle\right|^{2}=\|x\|^{2}-\sum_{i \in F}\left|\left\langle x, e_{i}\right\rangle\right|^{2},
$$

from which we easily deduce (2.2).

The following reverse of Bessel's inequality holds.

TheOREM 3. Let $\left\{e_{i}\right\}_{i \in I}$ be a family of orthornormal vectors in $H, F$ a finite part of $I, \phi_{i}, \Phi_{i}, i \in I$ real or complex numbers. Assume a $x \in H$. If either 
(i) $\operatorname{Re}\left\langle\sum_{i \in F} \Phi_{i} e_{i}-x, x-\sum_{i \in F} \phi_{i} e_{i}\right\rangle \geqslant 0$; or, equivalently,

(ii) $\left\|x-\sum_{i \in F}\left(\phi_{i}+\Phi_{i}\right) / 2 e_{i}\right\| \leqslant\left(\sum_{i \in F}\left|\Phi_{i}-\phi_{i}\right|^{2}\right)^{1 / 2} / 2$;

holds, then the following reverse of Bessel's inequality

$$
\begin{aligned}
0 & \leqslant\|x\|^{2}-\sum_{i \in F}\left|\left\langle x, e_{i}\right\rangle\right|^{2} \\
& \leqslant \frac{1}{4} \sum_{i \in F}\left|\Phi_{i}-\phi_{i}\right|^{2}-\sum_{i \in F}\left|\frac{\phi_{i}+\Phi_{i}}{2}-\left\langle x, e_{i}\right\rangle\right|^{2} \\
& \leqslant \frac{1}{4} \sum_{i \in F}\left|\Phi_{i}-\phi_{i}\right|^{2}
\end{aligned}
$$

is valid.

The constant $1 / 4$ is best possible in both inequalities.

Proof: Firstly, we observe that for $y, a, A \in H$, the following are equivalent

$$
\operatorname{Re}\langle A-y, y-a\rangle \geqslant 0
$$

and

$$
\left\|y-\frac{a+A}{2}\right\| \leqslant \frac{1}{2}\|A-a\| .
$$

Now, for $a=\sum_{i \in F} \phi_{i} e_{i}, A=\sum_{i \in F} \Phi_{i} e_{i}$, we have

$$
\begin{aligned}
\|A-a\| & =\left\|\sum_{i \in F}\left(\Phi_{i}-\phi_{i}\right) e_{i}\right\|=\left(\left\|\sum_{i \in F}\left(\Phi_{i}-\phi_{i}\right) e_{i}\right\|^{2}\right)^{1 / 2} \\
& =\left(\sum_{i \in F}\left|\Phi_{i}-\phi_{i}\right|^{2}\left\|e_{i}\right\|^{2}\right)^{1 / 2}=\left(\sum_{i \in F}\left|\Phi_{i}-\phi_{i}\right|^{2}\right)^{1 / 2}
\end{aligned}
$$

giving, for $y=x$, the desired equivalence.

Now, if we apply Lemma 1 for $\lambda_{i}=\left(\phi_{i}+\Phi_{i}\right) / 2$ and

$$
r:=\frac{1}{2}\left(\sum_{i \in F}\left|\Phi_{i}-\phi_{i}\right|^{2}\right)^{1 / 2}
$$

we deduce the first inequality in (2.4).

Let us prove that $1 / 4$ is best possible in the second inequality in (2.4). Assume that there is a $c>0$ such that

$$
0 \leqslant\|x\|^{2}-\sum_{i \in F}\left|\left\langle x, e_{i}\right\rangle\right|^{2} \leqslant c \sum_{i \in F}\left|\Phi_{i}-\phi_{i}\right|^{2}-\sum_{i \in F}\left|\frac{\phi_{i}+\Phi_{i}}{2}-\left\langle x, e_{i}\right\rangle\right|^{2}
$$


provided that $\phi_{i}, \Phi_{i}, x$ and $F$ satisfy (i) and (ii).

We choose $F=\{1\}, e_{1}=e=(1 / \sqrt{2},(1 / \sqrt{2})) \in \mathbb{R}^{2}, x=\left(x_{1}, x_{2}\right) \in \mathbb{R}^{2}, \Phi_{1}=\Phi$ $=m>0, \phi_{1}=\phi=-m, H=\mathbb{R}^{2}$ to get from (2.7) that

$$
\begin{aligned}
0 & \leqslant x_{1}^{2}+x_{2}^{2}-\frac{\left(x_{1}+x_{2}\right)^{2}}{2} \\
& \leqslant 4 \mathrm{~cm}^{2}-\frac{\left(x_{1}+x_{2}\right)^{2}}{2}
\end{aligned}
$$

provided

$$
\begin{aligned}
0 & \leqslant\langle m e-x, x+m e\rangle \\
& =\left(\frac{m}{\sqrt{2}}-x_{1}\right)\left(x_{1}+\frac{m}{\sqrt{2}}\right)+\left(\frac{m}{\sqrt{2}}-x_{2}\right)\left(\dot{x}_{2}+\frac{m}{\sqrt{2}}\right) .
\end{aligned}
$$

From (2.8) we get

$$
x_{1}^{2}+x_{2}^{2} \leqslant 4 c m^{2}
$$

provided (2.9) holds.

If we choose $x_{1}=m / \sqrt{2}, x_{2}=-m / \sqrt{2}$, then (2.9) is satisfied and by (2.10) we get $m^{2} \leqslant 4 \mathrm{~cm}^{2}$, giving $c \geqslant 1 / 4$.

REMARK 2. If $F=\{1\}, e_{1}=1$, $\|e\|=1$ and for $\phi, \Phi \in \mathbb{K}$ and $x \in H$ one has either

$$
\operatorname{Re}\langle\Phi e-x, x-\phi e\rangle \geqslant 0
$$

or, equivalently,

$$
\left\|x-\frac{\phi+\Phi}{2} e\right\| \leqslant \frac{1}{2}|\Phi-\phi|
$$

then

$$
\begin{aligned}
0 & \leqslant\|x\|^{2}-|\langle x, e\rangle|^{2} \\
& \leqslant \frac{1}{4}|\Phi-\phi|^{2}-\left|\frac{\phi+\Phi}{2}-\langle x, e\rangle\right|^{2} \leqslant \frac{1}{4}|\Phi-\phi|^{2} .
\end{aligned}
$$

The constant $1 / 4$ is best possible in both inequalities.

REMARK 3. It is important to compare the bounds provided by Corollary 1 and Theorem 3.

For this purpose, consider

$$
B_{1}(x, e, \phi, \Phi):=\frac{1}{4}(\Phi-\phi)^{2}-\langle\Phi e-x, x-\phi e\rangle
$$

and

$$
B_{2}(x, e, \phi, \Phi):=\frac{1}{4}(\Phi-\phi)^{2}-\left(\frac{\phi+\Phi}{2}-\langle x, e\rangle\right)^{2}
$$


where $H$ is a real inner product, $e \in H,\|e\|=1, x \in H, \phi, \Phi \in \mathbb{R}$ with $\langle\Phi e-x, x-\phi e\rangle \geqslant 0$, or equivalently,

$$
\left\|x-\frac{\phi+\Phi}{2} e\right\| \leqslant \frac{1}{2}|\Phi-\phi| .
$$

If we choose $\phi=-1, \Phi=1$, then we have

$$
\begin{aligned}
& B_{1}(x, e)=1-\langle e-x, x+e\rangle=1-\left(\|e\|^{2}-\|x\|^{2}\right)=\|x\|^{2}, \\
& B_{2}(x, e)=1-\langle x, e\rangle^{2},
\end{aligned}
$$

provided $\|x\| \leqslant 1$.

Choose $x=k e$, with $0<k \leqslant 1$. Then we get

$$
B_{1}(k)=k^{2}, \quad B_{2}(k)=1-k^{2},
$$

which shows that $B_{1}(k)>B_{2}(k)$ if $0<k<\sqrt{2} / 2$ and $B_{1}(k)<B_{2}(k)$ if $\sqrt{2} / 2<k \leqslant 1$.

We may state the following proposition.

Proposition 1. Let $\left\{e_{i}\right\}_{i \in I}$ be a family of orthornormal vectors in $H, F$ a finite part of $I, \phi_{i}, \Phi_{i} \in \mathbb{K}(i \in F)$. If $x \in H$ either satisfies (i), or, equivalently, (ii) of Theorem 3, then the upper bounds

$$
\begin{aligned}
& B_{1}(x, e, \phi, \Phi, F):=\frac{1}{4} \sum_{i \in F}\left|\Phi_{i}-\phi_{i}\right|^{2}-\operatorname{Re}\left\langle\sum_{i \in F} \Phi_{i} e_{i}-x, x-\sum_{i \in F} \phi_{i} e_{i}\right\rangle, \\
& B_{2}(x, e, \phi, \Phi, F):=\frac{1}{4} \sum_{i \in F}\left|\Phi_{i}-\phi_{i}\right|^{2}-\sum_{i \in F}\left|\frac{\phi_{i}+\Phi_{i}}{2}-\left\langle x, e_{i}\right\rangle\right|^{2},
\end{aligned}
$$

for the Bessel's difference $B_{s}(x, \mathbf{e}, F):=\|x\|^{2}-\sum_{i \in F}\left|\left\langle x, e_{i}\right\rangle\right|^{2}$, cannot be compared in general.

\section{A Refinement of the Grüss Inequality}

The following result holds.

THEOREM 4. Let $\left\{e_{i}\right\}_{i \in I}$ be a family of orthornormal vectors in $H, F$ a finite part of $I, \phi_{i}, \Phi_{i}, \gamma_{i}, \Gamma_{i} \in \mathbb{K}, i \in F$ and $x, y \in H$. If either

$$
\begin{aligned}
& \operatorname{Re}\left\langle\sum_{i \in F} \Phi_{i} e_{i}-x, x-\sum_{i \in F} \phi_{i} e_{i}\right\rangle \geqslant 0, \\
& \operatorname{Re}\left\langle\sum_{i \in F} \Gamma_{i} e_{i}-y, y-\sum_{i \in F} \gamma_{i} e_{i}\right\rangle \geqslant 0
\end{aligned}
$$

or, equivalently,

$$
\begin{aligned}
\left\|x-\sum_{i \in F} \frac{\Phi_{i}+\phi_{i}}{2} e_{i}\right\| & \leqslant \frac{1}{2}\left(\sum_{i \in F}\left|\Phi_{i}-\phi_{i}\right|^{2}\right)^{1 / 2}, \\
\left\|y-\sum_{i \in F} \frac{\Gamma_{i}+\gamma_{i}}{2} e_{i}\right\| & \leqslant \frac{1}{2}\left(\sum_{i \in F}\left|\Gamma_{i}-\gamma_{i}\right|^{2}\right)^{1 / 2},
\end{aligned}
$$


hold, then we have the inequalities

$$
\begin{aligned}
0 \leqslant & \left|\langle x, y\rangle-\sum_{i \in F}\left\langle x, e_{i}\right\rangle\left\langle e_{i}, y\right\rangle\right| \\
\leqslant & \frac{1}{4}\left(\sum_{i \in F}\left|\Phi_{i}-\phi_{i}\right|^{2}\right)^{1 / 2} \cdot\left(\sum_{i \in F}\left|\Gamma_{i}-\gamma_{i}\right|^{2}\right)^{1 / 2} \\
& \quad-\sum_{i \in F}\left|\frac{\Phi_{i}+\phi_{i}}{2}-\left\langle x, e_{i}\right\rangle\right|\left|\frac{\Gamma_{i}+\gamma_{i}}{2}-\left\langle y, e_{i}\right\rangle\right| \\
\leqslant & \frac{1}{4}\left(\sum_{i \in F}\left|\Phi_{i}-\phi_{i}\right|^{2}\right)^{1 / 2} \cdot\left(\sum_{i \in F}\left|\Gamma_{i}-\gamma_{i}\right|^{2}\right)^{1 / 2} \cdot
\end{aligned}
$$

The constant $1 / 4$ is best possible.

Proof: Using Schwarz's inequality in the inner product space $(H,\langle\cdot, \cdot\rangle)$ one has

$$
\left|\left\langle x-\sum_{i \in F}\left\langle x, e_{i}\right\rangle e_{i}, y-\sum_{i \in F}\left\langle y, e_{i}\right\rangle e_{i}\right\rangle\right|^{2} \leqslant\left\|x-\sum_{i \in F}\left\langle x, e_{i}\right\rangle e_{i}\right\|^{2}\left\|y-\sum_{i \in F}\left\langle y, e_{i}\right\rangle e_{i}\right\|^{2}
$$

and since a simple calculation shows that

$$
\left\langle x-\sum_{i \in F}\left\langle x, e_{i}\right\rangle e_{i}, y-\sum_{i \in F}\left\langle y, e_{i}\right\rangle e_{i}\right\rangle=\langle x, y\rangle-\sum_{i \in F}\left\langle x, e_{i}\right\rangle\left\langle e_{i}, y\right\rangle
$$

and

$$
\left\|x-\sum_{i \in F}\left\langle x, e_{i}\right\rangle e_{i}\right\|^{2}=\|x\|^{2}-\sum_{i \in F}\left|\left\langle x, e_{i}\right\rangle\right|^{2}
$$

for any $x, y \in H$, then by (3.4) and by the reverse of Bessel's inequality in Theorem 3, we have

$$
\begin{aligned}
\left|\langle x, y\rangle-\sum_{i \in F}\left\langle x, e_{i}\right\rangle\left\langle e_{i}, y\right\rangle\right|^{2} & \leqslant\left(\|x\|^{2}-\sum_{i \in F}\left|\left\langle x, e_{i}\right\rangle\right|^{2}\right)\left(\|y\|^{2}-\sum_{i \in F}\left|\left\langle y, e_{i}\right\rangle\right|^{2}\right) \\
& \leqslant\left[\frac{1}{4} \sum_{i \in F}\left|\Phi_{i}-\phi_{i}\right|^{2}-\sum_{i \in F}\left|\frac{\Phi_{i}+\phi_{i}}{2}-\left\langle x, e_{i}\right\rangle\right|^{2}\right] \\
\times & \times\left[\frac{1}{4} \sum_{i \in F}\left|\Gamma_{i}-\gamma_{i}\right|^{2}-\sum_{i \in F}\left|\frac{\Gamma_{i}+\gamma_{i}}{2}-\left\langle y, e_{i}\right\rangle\right|^{2}\right] \\
& :=K .
\end{aligned}
$$

Recall Aczél's inequality for real numbers, that is, we recall that

$$
\left(a^{2}-\sum_{i \in F} a_{i}^{2}\right)\left(b^{2}-\sum_{i \in F} b_{i}^{2}\right) \leqslant\left(a b-\sum_{i \in F} a_{i} b_{i}\right)^{2}
$$


provided that $a, b, a_{i}, b_{i}>0, i \in F$, and either $a^{2}-\sum_{i \in F} a_{i}^{2}>0$ or $b^{2}-\sum_{i \in F} b_{i}^{2}>0$. So we may state that

$$
\begin{aligned}
& K \leqslant\left[\frac{1}{4}\left(\sum_{i \in F}\left|\Phi_{i}-\phi_{i}\right|^{2}\right)^{1 / 2} \cdot\left(\sum_{i \in F}\left|\Gamma_{i}-\gamma_{i}\right|^{2}\right)^{1 / 2}\right. \\
&\left.-\sum_{i \in F}\left|\frac{\Phi_{i}+\phi_{i}}{2}-\left\langle x, e_{i}\right\rangle\right|\left|\frac{\Gamma_{i}+\gamma_{i}}{2}-\left\langle y, e_{i}\right\rangle\right|\right]^{2}
\end{aligned}
$$

Using (3.5) and (3.7) we conclude that

$$
\begin{aligned}
\left|\langle x, y\rangle-\sum_{i \in F}\left\langle x, e_{i}\right\rangle\left\langle e_{i}, y\right\rangle\right|^{2} \leqslant & {\left[\frac{1}{4}\left(\sum_{i \in F}\left|\Phi_{i}-\phi_{i}\right|^{2}\right)^{1 / 2} \cdot\left(\sum_{i \in F}\left|\Gamma_{i}-\gamma_{i}\right|^{2}\right)^{1 / 2}\right.} \\
& \left.-\sum_{i \in F}\left|\frac{\Phi_{i}+\phi_{i}}{2}-\left\langle x, e_{i}\right\rangle\right|\left|\frac{\Gamma_{i}+\gamma_{i}}{2}-\left\langle y, e_{i}\right\rangle\right|\right]^{2} .
\end{aligned}
$$

Taking the square root in (3.8) and taking into account that the quantity in the last square brackets is nonnegative (use for example (2.4) and the Cauchy-Bunyakovsky-Schwarz's inequality for real numbers), we deduce the second inequality in (3.3).

The fact that $1 / 4$ is the best possible constant follows by Theorem 3 and we omit the details.

The following corollary may be stated.

Corollary 2'. Let $e \in H,\|e\|=1, \phi, \Phi, \gamma, \Gamma \in \mathbb{K}$ and $x, y \in H$ such that either

$$
\operatorname{Re}\langle\Phi e-x, x-\phi e\rangle \geqslant 0 \text { and } \operatorname{Re}\langle\Gamma e-y, y-\gamma e\rangle \geqslant 0
$$

or, equivalently,

$$
\left\|x-\frac{\phi+\Phi}{2} e\right\| \leqslant \frac{1}{2}|\Phi-\phi|, \quad\left\|y-\frac{\gamma+\Gamma}{2} e\right\| \leqslant \frac{1}{2}|\Gamma-\gamma| .
$$

Then we have the following refinement of Grüss' inequality

$$
\begin{aligned}
0 & \leqslant|\langle x, y\rangle-\langle x, e\rangle\langle e, y\rangle| \\
& \leqslant \frac{1}{4}|\Phi-\phi||\Gamma-\gamma|-\left|\frac{\phi+\Phi}{2}-\langle x, e\rangle\right|\left|\frac{\gamma+\Gamma}{2}-\langle y, e\rangle\right| \\
& \leqslant \frac{1}{4}|\Phi-\phi||\Gamma-\gamma| .
\end{aligned}
$$

The constant $1 / 4$ is best possible in both inequalities.

\section{Some Companion InEQualities}

The following companion of the Grüss inequality also holds. 
THEOREM 5. Let $\left\{e_{i}\right\}_{i \in I}$ be a family of orthornormal vectors in $H, F$ a finite part of $I$ and $\phi_{i}, \Phi_{i} \in \mathbb{K}, i \in F, x, y \in H$ and $\lambda \in(0,1)$, such that either

$$
\operatorname{Re}\left\langle\sum_{i \in F} \Phi_{i} e_{i}-(\lambda x+(1-\lambda) y), \lambda x+(1-\lambda) y-\sum_{i \in F} \phi_{i} e_{i}\right\rangle \geqslant 0
$$

or, equivalently,

$$
\left\|\lambda x+(1-\lambda) y-\sum_{i \in F} \frac{\Phi_{i}+\phi_{i}}{2} \cdot e_{i}\right\| \leqslant \frac{1}{2}\left(\sum_{i \in F}\left|\Phi_{i}-\phi_{i}\right|^{2}\right)^{1 / 2},
$$

holds. Then we have the inequality

$$
\begin{aligned}
\operatorname{Re}\left[\langle x, y\rangle-\sum_{i \in F}\left\langle x, e_{i}\right\rangle\left\langle e_{i}, y\right\rangle\right] \leqslant & \frac{1}{16} \cdot \frac{1}{\lambda(1-\lambda)} \sum_{i \in F}\left|\Phi_{i}-\phi_{i}\right|^{2} \\
& -\frac{1}{4} \frac{1}{\lambda(1-\lambda)} \sum_{i \in F}\left|\frac{\Phi_{i}+\phi_{i}}{2}-\left\langle\lambda x+(1-\lambda) y, e_{i}\right\rangle\right|^{2} \\
& \leqslant \frac{1}{16} \cdot \frac{1}{\lambda(1-\lambda)} \sum_{i \in F}\left|\Phi_{i}-\phi_{i}\right|^{2}
\end{aligned}
$$

The constant $1 / 16$ is the best possible constant in (4.3) in the sense that it cannot be replaced by a smaller constant.

Proof: We know that for any $z, u \in H$, one has

$$
\operatorname{Re}\langle z, u\rangle \leqslant \frac{1}{4}\|z+u\|^{2} \text {. }
$$

Then for any $a, b \in H$ and $\lambda \in(0,1)$ one has

$$
\operatorname{Re}\langle a, b\rangle \leqslant \frac{1}{4 \lambda(1-\lambda)}\|\lambda a+(1-\lambda) b\|^{2} .
$$

Since

$$
\langle x, y\rangle-\sum_{i \in F}\left\langle x, e_{i}\right\rangle\left\langle e_{i}, y\right\rangle=\left\langle x-\sum_{i \in F}\left\langle x, e_{i}\right\rangle e_{i}, y-\sum_{i \in F}\left\langle y, e_{i}\right\rangle e_{i}\right\rangle,
$$

for any $x, y \in H$, then, by (4.4), we get

$$
\begin{aligned}
& \operatorname{Re}\left[\langle x, y\rangle-\sum_{i \in F}\left\langle x, e_{i}\right\rangle\left\langle e_{i}, y\right\rangle\right] \\
& \quad=\operatorname{Re}\left[\left\langle x-\sum_{i \in F}\left\langle x, e_{i}\right\rangle e_{i}, y-\sum_{i \in F}\left\langle y, e_{i}\right\rangle e_{i}\right\rangle\right] \\
& \quad \leqslant \frac{1}{4 \lambda(1-\lambda)}\left\|\lambda\left(x-\sum_{i \in F}\left\langle x, e_{i}\right\rangle e_{i}\right)+(1-\lambda)\left(y-\sum_{i \in F}\left(y, e_{i}\right\rangle e_{i}\right)\right\|^{2}
\end{aligned}
$$




$$
\begin{aligned}
& =\frac{1}{4 \lambda(1-\lambda)}\left\|\lambda x+(1-\lambda) y-\sum_{i \in F}\left\langle\lambda x+(1-\lambda) y, e_{i}\right\rangle e_{i}\right\|^{2} \\
& =\frac{1}{4 \lambda(1-\lambda)}\left[\|\lambda x+(1-\lambda) y\|^{2}-\sum_{i \in F}\left|\left\langle\lambda x+(1-\lambda) y, e_{i}\right\rangle\right|^{2}\right] .
\end{aligned}
$$

If we apply the reverse of Bessel's inequality in Theorem 3 for $\lambda x+(1-\lambda) y$, we may state that

$$
\begin{aligned}
\| \lambda x+(1 & -\lambda) y \|^{2}-\sum_{i \in F}\left|\left\langle\lambda x+(1-\lambda) y, e_{i}\right\rangle\right|^{2} \\
& \leqslant \frac{1}{4} \sum_{i \in F}\left|\Phi_{i}-\phi_{i}\right|^{2}-\sum_{i \in F}\left|\frac{\Phi_{i}+\phi_{i}}{2}-\left\langle\lambda x+(1-\lambda) y, e_{i}\right\rangle\right|^{2} \\
& \leqslant \frac{1}{4} \sum_{i \in F}\left|\Phi_{i}-\phi_{i}\right|^{2} .
\end{aligned}
$$

Now, by making use of (4.5) and (4.6), we deduce (4.3).

The fact that $1 / 16$ is the best possible constant in (4.3) follows by the fact that if in (4.1) we choose $x=y$, then it becomes (i) of Theorem 3 , implying for $\lambda=1 / 2$ the inequality (2.4), for which, we have shown that $1 / 4$ is the best constant.

REMARK 4. In practical applications we may use only the inequality between the first and the last term in (4.3).

REMARK 5. If in Theorem 5, we choose $\lambda=1 / 2$, then we get

$$
\begin{aligned}
\operatorname{Re}[\langle x, y\rangle & \left.-\sum_{i \in F}\left\langle x, e_{i}\right\rangle\left\langle e_{i}, y\right\rangle\right] \\
& \leqslant \frac{1}{4} \sum_{i \in F}\left|\Phi_{i}-\phi_{i}\right|^{2}-\sum_{i \in F}\left|\frac{\Phi_{i}+\phi_{i}}{2}-\left\langle\frac{x+y}{2}, e_{i}\right\rangle\right|^{2} \\
& \leqslant \frac{1}{4} \sum_{i \in F}\left|\Phi_{i}-\phi_{i}\right|^{2},
\end{aligned}
$$

provided

$$
\operatorname{Re}\left\langle\sum_{i \in F} \Phi_{i} e_{i}-\frac{x+y}{2}, \frac{x+y}{2}-\sum_{i \in F} \phi_{i} e_{i}\right\rangle \geqslant 0
$$

or, equivalently,

$$
\left\|\frac{x+y}{2}-\sum_{i \in F} \frac{\Phi_{i}+\phi_{i}}{2} \cdot e_{i}\right\| \leqslant \frac{1}{2}\left(\sum_{i \in F}\left|\Phi_{i}-\phi_{i}\right|^{2}\right)^{1 / 2} .
$$

We note that (4.7) is a refinement of the corresponding result in [3]. 
COROLlary 3. With the assumptions of Theorem 5 and if

$$
\operatorname{Re}\left\langle\sum_{i \in F} \Phi_{i} e_{i}-(\lambda x \pm(1-\lambda) y), \lambda x \pm(1-\lambda) y-\sum_{i \in F} \phi_{i} e_{i}\right\rangle \geqslant 0
$$

or, equivalently

$$
\left\|\lambda x \pm(1-\lambda) y-\sum_{i \in F} \frac{\Phi_{i}+\phi_{i}}{2} \cdot e_{i}\right\| \leqslant \frac{1}{2}\left(\sum_{i \in F}\left|\Phi_{i}-\phi_{i}\right|^{2}\right)^{1 / 2},
$$

then we have the inequality

$$
\left|\operatorname{Re}\left[\langle x, y\rangle-\sum_{i \in F}\left\langle x, e_{i}\right\rangle\left\langle e_{i}, y\right\rangle\right]\right| \leqslant \frac{1}{16} \cdot \frac{1}{\lambda(1-\lambda)} \sum_{i \in F}\left|\Phi_{i}-\phi_{i}\right|^{2} .
$$

The constant 1/16 is best possible in (4.10).

REMARK 6. If $H$ is a real inner product space and $m_{i}, M_{i} \in \mathbb{R}$ with the property

$$
\left\langle\sum_{i \in F} M_{i} e_{i}-(\lambda x \pm(1-\lambda) y), \lambda x \pm(1-\lambda) y-\sum_{i \in F} m_{i} e_{i}\right\rangle \geqslant 0
$$

or, equivalently,

$$
\left\|\lambda x \pm(1-\lambda) y-\sum_{i \in F} \frac{M_{i}+m_{i}}{2} \cdot e_{i}\right\| \leqslant \frac{1}{2}\left[\sum_{i \in F}\left(M_{i}-m_{i}\right)^{2}\right]^{1 / 2},
$$

then we have the Grüss type inequality

$$
\left|\langle x, y\rangle-\sum_{i \in F}\left\langle x, e_{i}\right\rangle\left\langle e_{i}, y\right\rangle\right| \leqslant \frac{1}{16} \cdot \frac{1}{\lambda(1-\lambda)} \sum_{i \in F}\left(M_{i}-m_{i}\right)^{2} .
$$

\section{INTEgRal INEQUaLities}

Let $(\Omega, \Sigma, \mu)$ be a measure space consisting of a set $\Omega, \Sigma$ a $\sigma$-algebra of subsets and $\mu$ a countably additive and positive measure on $\Sigma$ with values in $\mathbb{R} \cup\{\infty\}$. Let $\rho \geqslant 0$ be a $\mu$-measurable function on $\Omega$. Denote by $L_{\rho}^{2}(\Omega, \mathbb{K})$ the Hilbert space of all real or complex valued functions defined on $\Omega$ such that

$$
\int_{\Omega} \rho(s)|f(s)|^{2} d \mu(s)<\infty .
$$

Consider the family $\left\{f_{i}\right\}_{i \in I}$ of functions in $L_{\rho}^{2}(\Omega, \mathbb{K})$ with the properties that

$$
\int_{\Omega} \rho(s) f_{i}(s) \overline{f_{j}}(s) d \mu(s)=\delta_{i j}, \quad i, j \in I,
$$

where $\delta_{i j}$ is 0 if $i \neq j$ and $\delta_{i j}=1$ if $i=j .\left\{f_{i}\right\}_{i \in J}$ is an orthornormal family in $L_{\rho}^{2}(\Omega, \mathbb{K})$.

The following proposition holds. 
PROPOS ITION 2. Let $\left\{f_{i}\right\}_{i \in I}$ be an orthornormal family of functions in $L_{\rho}^{2}(\Omega, \mathbb{K})$, $F$ a finite subset of $I, \phi_{i}, \Phi_{i} \in \mathbb{K}(i \in F)$ and $f \in L_{\rho}^{2}(\Omega, \mathbb{K})$, so that either

$$
\int_{\Omega} \rho(s) \operatorname{Re}\left[\left(\sum_{i \in F} \Phi_{i} f_{i}(s)-f(s)\right)\left(\bar{f}(s)-\sum_{i \in F} \bar{\phi}_{i} \bar{f}_{i}(s)\right)\right] d \mu(s) \geqslant 0
$$

or, equivalently,

$$
\int_{\Omega} \rho(s)\left|f(s)-\sum_{i \in F} \frac{\Phi_{i}+\phi_{i}}{2} \cdot f_{i}(s)\right|^{2} d \mu(s) \leqslant \frac{1}{4} \sum_{i \in F}\left|\Phi_{i}-\phi_{i}\right|^{2}
$$

hold. Then we have the inequality

$$
\begin{aligned}
0 & \leqslant \int_{\Omega} \rho(s)|f(s)|^{2} d \mu(s)-\sum_{i \in F}\left|\int_{\Omega} \rho(s) f(s) \overline{f_{i}}(s) d \mu(s)\right|^{2} \\
& \leqslant \frac{1}{4} \sum_{i \in F}\left|\Phi_{i}-\phi_{i}\right|^{2}-\sum_{i \in F}\left|\frac{\Phi_{i}+\phi_{i}}{2}-\int_{\Omega} \rho(s) f(s) \overline{f_{i}}(s) d \mu(s)\right|^{2} \\
& \leqslant \frac{1}{4} \sum_{i \in F}\left|\Phi_{i}-\phi_{i}\right|^{2} .
\end{aligned}
$$

The constant $1 / 4$ is best possible in both inequalities.

The proof follows by Theorem 3 applied for the Hilbert space $L_{\rho}^{2}(\Omega, \mathbb{K})$ and the orthornormal family $\left\{f_{i}\right\}_{i \in I}$.

The following Grüss type inequality also holds.

Proposition 3. Let $\left\{f_{i}\right\}_{i \in I}$ and $F$ be as in Proposition 2. If $\phi_{i}, \Phi_{i}, \gamma_{i}, \Gamma_{i} \in \mathbb{K}$ $(i \in F)$ and $f, g \in L_{\rho}^{2}(\Omega, \mathbb{K})$ so that either

$$
\begin{gathered}
\int_{\Omega} \rho(s) \operatorname{Re}\left[\left(\sum_{i \in F} \Phi_{i} f_{i}(s)-f(s)\right)\left(\bar{f}(s)-\sum_{i \in F} \overline{\phi_{i}} \overline{f_{i}}(s)\right)\right] d \mu(s) \geqslant 0 \\
\int_{\Omega} \rho(s) \operatorname{Re}\left[\left(\sum_{i \in F} \Gamma_{i} f_{i}(s)-g(s)\right)\left(\bar{g}(s)-\sum_{i \in F} \overline{\gamma_{i}} \overline{f_{i}}(s)\right)\right] d \mu(s) \geqslant 0
\end{gathered}
$$

or, equivalently,

$$
\begin{gathered}
\int_{\Omega} \rho(s)\left|f(s)-\sum_{i \in F} \frac{\Phi_{i}+\phi_{i}}{2} f_{i}(s)\right|^{2} d \mu(s) \leqslant \frac{1}{4} \sum_{i \in F}\left|\Phi_{i}-\phi_{i}\right|^{2} \\
\int_{\Omega} \rho(s)\left|g(s)-\sum_{i \in F} \frac{\Gamma_{i}+\gamma_{i}}{2} f_{i}(s)\right|^{2} d \mu(s) \leqslant \frac{1}{4} \sum_{i \in F}\left|\Gamma_{i}-\gamma_{i}\right|^{2}
\end{gathered}
$$


hold, then we have the inequalities

$$
\begin{aligned}
& \left|\int_{\Omega} \rho(s) f(s) \overline{g(s)} d \mu(s)-\sum_{i \in F} \int_{\Omega} \rho(s) f(s) \overline{f_{i}}(s) d \mu(s) \int_{\Omega} \rho(s) f_{i}(s) \overline{g(s)} d \mu(s)\right| \\
& \quad \leqslant \frac{1}{4}\left(\sum_{i \in F}\left|\Phi_{i}-\phi_{i}\right|^{2}\right)^{1 / 2}\left(\sum_{i \in F}\left|\Gamma_{i}-\gamma_{i}\right|^{2}\right)^{1 / 2} \\
& \quad-\sum_{i \in F}\left|\frac{\Phi_{i}+\phi_{i}}{2}-\int_{\Omega} \rho(s) f(s) \overline{f_{i}}(s) d \mu(s)\right|\left|\frac{\Gamma_{i}+\gamma_{i}}{2}-\int_{\Omega} \rho(s) g(s) \overline{f_{i}}(s) d \mu(s)\right| \\
& \quad \leqslant \frac{1}{4}\left(\sum_{i \in F}\left|\Phi_{i}-\phi_{i}\right|^{2}\right)^{1 / 2}\left(\sum_{i \in F}\left|\Gamma_{i}-\gamma_{i}\right|^{2}\right)^{1 / 2} .
\end{aligned}
$$

The constant $1 / 4$ is the best possible.

The proof follows by Theorem 4 and we omit the details.

REMARK 7. Similar results may be stated if one applies the inequalities in Section 4. We omit the details.

In the case of real spaces, the following corollaries provide much simpler sufficient conditions for the reverse of Bessel's inequality (5.5) or for the Grüss type inequality (5.8) to hold.

COROLlaRY 4. Let $\left\{f_{i}\right\}_{i \in I}$ be an orthornormal family of functions in the real Hilbert space $L_{\rho}^{2}(\Omega), F$ a finite part of $I, M_{i}, m_{i} \in \mathbb{R} \quad(i \in F)$ and $f \in L_{\rho}^{2}(\Omega)$ so that

$$
\sum_{i \in F} m_{i} f_{i}(s) \leqslant f(s) \leqslant \sum_{i \in F} M_{i} f_{i}(s) \text { for } \mu-\text { almost everywhere } s \in \Omega
$$

then we have the inequalities

$$
\begin{aligned}
0 & \leqslant \int_{\Omega} \rho(s) f^{2}(s) d \mu(s)-\sum_{i \in F}\left[\int_{\Omega} \rho(s) f(s) f_{i}(s) d \mu(s)\right]^{2} \\
& \leqslant \frac{1}{4} \sum_{i \in F}\left(M_{i}-m_{i}\right)^{2}-\sum_{i \in F}\left[\frac{M_{i}+m_{i}}{2}-\int_{\Omega} \rho(s) f(s) f_{i}(s) d \mu(s)\right]^{2} \\
& \leqslant \frac{1}{4} \sum_{i \in F}\left(M_{i}-m_{i}\right)^{2} .
\end{aligned}
$$

The constant $1 / 4$ is best possible.

COROLlary 5. Let $\left\{f_{i}\right\}_{i \in I}$ and $F$ be as in Corollary 4. If $M_{i}, m_{i}, N_{i}, n_{i} \in \mathbb{R}$ $(i \in F)$ and $f, g \in L_{\rho}^{2}(\Omega)$ such that

$$
\sum_{i \in F} m_{i} f_{i}(s) \leqslant f(s) \leqslant \sum_{i \in F} M_{i} f_{i}(s)
$$

and

$$
\sum_{i \in F} n_{i} f_{i}(s) \leqslant g(s) \leqslant \sum_{i \in F} N_{i} f_{i}(s) \text { for } \mu \text { - almost everywhere } s \in \Omega
$$


then we have the inequalities

$$
\begin{aligned}
& \left|\int_{\Omega} \rho(s) f(s) g(s) d \mu(s)-\sum_{i \in F} \int_{\Omega} \rho(s) f(s) f_{i}(s) d \mu(s) \int_{\Omega} \rho(s) g(s) f_{i}(s) d \mu(s)\right| \\
& \leqslant \frac{1}{4}\left(\sum_{i \in F}\left(M_{i}-m_{i}\right)^{2}\right)^{1 / 2}\left(\sum_{i \in F}\left(N_{i}-n_{i}\right)^{2}\right)^{1 / 2}-\sum_{i \in F}\left|\frac{M_{i}+m_{i}}{2}-\int_{\Omega} \rho(s) f(s) f_{i}(s) d \mu(s)\right| \\
& \times\left|\frac{N_{i}+n_{i}}{2}-\int_{\Omega} \rho(s) g(s) f_{i}(s) d \mu(s)\right| \\
& \leqslant \frac{1}{4}\left(\sum_{i \in F}\left(M_{i}-m_{i}\right)^{2}\right)^{1 / 2}\left(\sum_{i \in F}\left(N_{i}-n_{i}\right)^{2}\right)^{1 / 2}
\end{aligned}
$$

\section{REFERENCES}

[1] S.S. Dragomir, 'A generalisation of Grüss' inequality in inner product spaces and applications', J. Math. Anal. Appl. 237 (1999), 74-82.

[2] S.S. Dragomir, 'Some Grüss' type inequalities in inner product spaces', J. Ineq. Pure Appl. Math. 4 (2003), 10. Article 42 [ONLINE http://jipam.vu.edu.au/v4n2/032_03.html].

[3] S.S. Dragomir, 'A counterpart of Bessel's inequality in inner product spaces and some Grüss type related results', RGMIA Res. Rep. Coll. 6 (2003). Supplement, Article 10 [ONLINE http://rgmia.vu.edu.au/v6(E) .html].

[4] N. Ujević, A new generalisation of Grüss inequality in inner product spaces, Math. Ineq. Appl. 6 (2003), 617-623.

School of Computer Science and Mathematics

Victoria University of Technology

PO Box 14428

MCMC 8001 Victoria

Australia

e-mail sever.dragomir@vu.edu.au

urladdrhttp://rgmia.vu.edu.au/SSDragomirWeb.html 